\title{
Electron injector based on thermionic RF modulated electron gun for particle accelerator applications
}

\author{
Liang Zhang, Senior Member, IEEE, Georgia Adam, Boris Militsyn, Wenlong He and Adrian W. Cross
}

\begin{abstract}
In this paper, the design and simulation of an electron injector based on a thermionic RF modulated electron gun for particle accelerator applications is presented. The electron gun is based on a gridded thermionic cathode with the geometry based on a Pierce-type configuration. Both theory and numerical simulation were used to explore the relationship between the bunch length and charge. The reasons for the pulse widening were also analyzed. The beam dynamics simulations showed that a minimum pulse length of 106 ps could be achieved with a bunch charge of $33 \mathrm{pC}$ when the driving $\mathrm{RF}$ frequency was $1.5 \mathrm{GHz}$. The average transverse emittance was about $17 \mathrm{~mm} \cdot \mathrm{mrad}$ from the particle-in-cell simulations. Operating at a higher RF frequency did not significantly reduce the micro-pulse length.
\end{abstract}

Index Terms - RF gun, thermionic electron gun, particle accelerators.

\section{INTRODUCTION}

Electron beam sources in radio-frequency (RF) electron guns which generate high peak current and low emittance electron beams in a particle accelerator are core to the operation of coherent radiation sources such as Free-electron lasers (FELs) [1] and medical linacs [2]. There are three main types of RF electron guns used as injectors, including thermionic cathodes [3], photocathodes [4], and field emission cathodes [5]. In thermionic RF guns, the cathode is heated and electrons are continuously emitted and accelerated during the half RF period. This results in a long electron beam pulse and a wide energy spread at the exit of the linac. The problem of energy spread and pulse duration control is solved by using photocathode RF guns, where a laser pulse is used to extract the electrons from the cathode material. Short laser pulses generate short electron bunches with higher current densities. However, the performance of photocathodes is limited by the availability of the high power laser. Another type of RF gun is based on field emission which uses a needle-shaped cathode placed in a high electric field but it is fragile and prone to damage by vaporization of the cathode tip when generating high current density electron pulses.

L. Zhang (liang.zhang@strath.ac.uk), G. Adam (georgia.adam@strath.ac. uk) and A. W. Cross (a.w.cross@strath.ac.uk) are with Department of Physics, SUPA, University of Strathclyde, Glasgow, G4 0NG, Scotland, UK and the Cockcroft Institute, Warrington Bank Quay. UK. B. Militsyn (boris.militsyn @ stfc.ac.uk) is with the Cockcroft Institute, Sci-Tech Daresbury, and the ASTeC, STFC Daresbury Laboratory, Sci-Tech Daresbury, Keckwick Lane, Daresbury, Cheshire, WA4 4AD, United Kingdom. W. He
The photocathode can generate the ideal electron bunch for the accelerator. It has the advantages of large current density, short pulse and low intrinsic emittance to match every acceleration RF bucket, and high quality producing low emittance electron beams [4, 6-9]. However, there are challenges involved in operating photocathodes such as invacuum photocathode replacement systems to improve lifetime, requirements of ultra-high vacuum, as well as use of a high power drive laser which limits its applications to particle accelerators that require the very lowest emittance beams.

As a comparison, the thermionic cathode can produce high average power electron beams and has the advantages of a long lifetime, proven in many vacuum electronic devices which have operating lifetimes of longer than 100,000 hours. The thermionic cathode is also cost-effective and can produce large current densities of $10-100 \mathrm{~A} / \mathrm{cm}^{2}$ with increased lifetime achieved the lower the current density due to a more modest emitter operating temperature [10]. A thermionic RF gun is compact in structure and is relatively simple to operate. It also can be operated at high repetition rates.

Normally a diode or triode type thermionic electron gun is used. In a diode-type cathode, the high voltage is applied between the cathode and the anode to produce a $\mathrm{CW}$ electron beam. The electrons that fill the acceleration RF bucket are accelerated. The electrons outside the acceleration RF bucket will either strike the acceleration cavity's wall or back bombard on the cathode surface. It can cause significant thermal issues and shorten the cathode's lifetime as well as producing stray Xray radiation.

The object of this paper is to investigate the modulation of the electron beam, produced by the thermionic cathode, to significantly improve the efficiency of electron beam acceleration. The diode-type cathode can be gated by switching on/off the high voltage power supply. However, its repetition rate is very low. A triode-type cathode which has an additional electrode between the cathode and anode can achieve a much higher repetition rate since the required drive voltage is much lower. It can be driven by an RF voltage to gate the electron

(wenlong.he@szu.edu.cn) is with College of Electronic Science and Technology, Shenzhen University, Shenzhen, 518060, China. This work is under the support of Science and Technology Facilities Council (STFC) U.K. research grant, ST/S002081/1. Georgia Adam would like to thank STFC, UK and TMD Technologies Ltd for providing the industrial Accelerators for Security Health and the Environment (ASHE) PhD case studentship. 
beam and has attracted a great deal of interest as an electron beam source in RF linacs [11-14] as the electron micro-pulses can be matched with the acceleration RF buckets as well as achieving control of the emitted electron beam.

The charge, length and emittance of the generated electron bunch are important parameters for the thermionic RF modulated gun used in linacs. In this paper, their relation was obtained from a simplified theoretical model and further studied by particle-in-cell simulation through the design of an RF gun for a linac [15]. Section II discusses the configuration of the RF gun as well as the RF cavity. The theoretical model of the RF gun and the relationship between the bunch charge and bunch length is presented in section III. Section IV describes the design process of an RF electron gun. The performance, including the relationship between the bunch charge and bunch length and the reasons for the widening of the bunch length is discussed in section $\mathrm{V}$.

\section{CONFigurations OF THE RF GUN}

The configuration of the electron gun with RF gating is shown in Fig. 1. The emitted electron beam is modulated by the $\mathrm{RF}$ voltage, applied between the cathode and the grid. The RF gun in Fig. 1(a) is placed at the entrance of the RF acceleration cells, and the electron beam will be directly accelerated by the electric field in the cells $[3,11,13,14]$. It has the advantage that the cathode structure is simple. However, since the cathode is also a part of the RF acceleration circuit, it is difficult to be demounted because it may change the cavity parameters, and it is hard to avoid RF heating of the grid. The configuration in Fig. 1(b) contains an additional DC acceleration section. It enables pre-acceleration of the electron beam and focusing of the emitted electrons to achieve a smaller radius and divergence since an optimal focusing electrode can be used [16]. Another advantage is the design of the cathode and the acceleration cells can be decoupled. The RF gun can be demountable to save the cost of replacing the whole linac as well as reducing maintenance. The electron gun in the linac requires long lifetime, low cost and needs to be easily maintained on a limited budget, hence a demountable capability can be very attractive. Therefore, the configuration in Fig. 1(b) is studied in this paper.

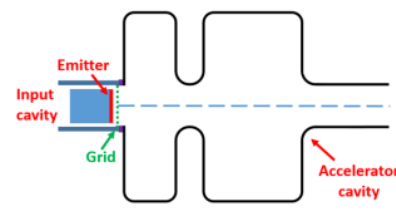

(a)

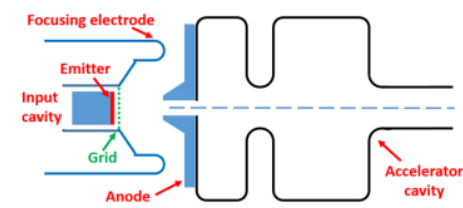

(b)
Fig. 1. The configuration of the RF gun, (a) without DC pre-acceleration, (b) with DC pre-acceleration.

The initial electron beam parameters of the proposed linac are listed in Table 1. The study was to design a thermionic cathode RF gated gun to meet the required beam parameters, and also to investigate the properties of the electron bunch, including the bunch charge, bunch length and emittance.

Table 1 the specifications of the linac

\begin{tabular}{|l|l|}
\hline Beam energy & $6 \mathrm{MeV}$ \\
\hline
\end{tabular}

\begin{tabular}{|l|l|}
\hline Acceleration frequency & $3 \mathrm{GHz}$ \\
\hline Repetition rate & $300 \mathrm{~Hz}$ \\
\hline RF pulse length & $7.5 \mathrm{us}$ \\
\hline RF gated frequency $(\mathrm{GHz})$ & 1.5 or 3.0 \\
\hline Bunch charge $Q(\mathrm{pc})$ & $33.3 @ 1.5 \mathrm{GHz}$ \\
& $16.7 @ 3.0 \mathrm{GHz}$ \\
\hline Bunch length $\tau(\mathrm{ps})$ & As short as possible \\
\hline Peak current $(\mathrm{A})$ & $\mathrm{Q} / \tau$ \\
\hline Pre-acceleration voltage $(\mathrm{kV})$ & 25 \\
\hline Beam radius $(\mathrm{mm})$ & $<2.5$ \\
\hline
\end{tabular}

\section{A THEORETICAL MODEL OF THE BUNCH LENGTH}

The RF voltage can be driven through a capacitively-loaded coaxial cavity. Its basic geometry is shown in Fig. 2(a) and its eigenfrequency $\omega_{0}$ can be estimated with

$$
\frac{1}{z_{0}} \cot (\beta L)-\omega_{0} C=0
$$

where $Z_{0}$ is the impedance, $\beta$ is the wavenumber, and the capacitance of the cavity $C=C_{1}+C_{2}=\frac{\varepsilon \pi a^{2}}{d}+4 \varepsilon a \ln \frac{b-a}{d}$. The eigenfrequency is more sensitive to the cavity length $L$, therefore it can be used for fine-tuning after the other dimensions are determined.

A practical structure of the input cavity for the RF electron gun is shown in Fig. 2(b) [17]. The parameters $a, b$ and $d$ are determined by the electron gun geometry. Its eigenfrequency can be tuned by the cavity length. Fig. 2(b) also shows its electric field pattern calculated using the 3D EM simulation software CST Microwave Studio at a resonance frequency of $1.5 \mathrm{GHz}$. In the simulation, the grid structure is simplified as a perfect electrical conductor (PEC) since the sizes of the grids are much smaller than the wavelength of the operating mode.

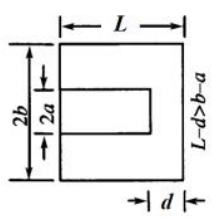

(a)

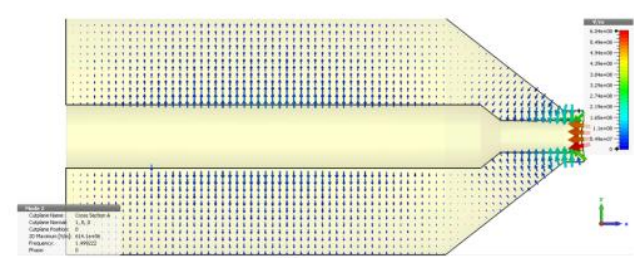

(b)
Fig. 2. The schematic of a coaxial input cavity (a) and the electric field pattern from the eigenmode simulation (b).

The thermionic electron gun can operate in the space charge limited or temperature limited regimes. The space charge limited current density follows the Child Langmuir law [18], which is determined by the field strength at the emitter surface, while the current density at the temperature limited regime follows with Richardson's law and it is mainly determined by the temperature at the emitter surface [19]. To be RF gated, the electron gun in the linac should operate at the space charge limited regime to totally switch off the electron beam. Also operating at lower temperatures and smaller current density, an electron gun with a long lifetime and low emittance is possible. The current density in the space charge limited emission regime applied with a static electric field is given by

$$
J=\frac{I}{S}=\frac{4 \varepsilon_{0}}{9} \sqrt{\frac{2 e}{m}} \frac{U_{\text {grid }}^{3 / 2}}{d^{2}} \quad\left(U_{\text {grid }}>0\right)
$$


where $U_{\text {grid }}$ is the total voltage across the cathode and the grid, $d$ is their distance, and $S$ is the area of the cathode. When driven by a RF voltage $U_{r f}$, the emitted current will also be modulated with the same frequency. However, when the frequency of RF voltage is high, and the pulse length of the electron beam is less than the transit time from the emitter to the grid, the classic Child Langmuir law will no longer be valid. The current density for a short current pulse can be obtained by applying a correction factor to the steady-state value of the classic Child Langmuir law, which is given by [20]

$$
J_{\text {short }}=2 \frac{1-\sqrt{1-3 X_{C L}^{2} / 4}}{X_{C L}^{3}} \mathrm{~J}
$$

where $X_{C L}=\tau / T_{C L} \leq 1$ is the normalized transit time and $T_{C L}=3 d / \sqrt{2 e U_{\text {grid }} / m}$ is the transit time from the emitter to the grid. For the thermionic RF gated gun studied in this paper, the transit time is $\sim 60 \mathrm{ps}$, and it is smaller than the pulse duration ( 100 ps). The classic Child Langmuir law is still valid.

Eq. 2 indicates that the current emits from the cathode at half of the RF cycle when $U_{\text {grid }}>0$. To control the emission time, a negative bias voltage $U_{\text {bias }}$ can be applied to stop the emission of the electrons, and the total grid voltage becomes

$$
U_{\text {grid }}=U_{r f} \cos (2 \pi f t-\theta)+U_{\text {bias }}
$$

Fig. 3 shows the typical waveforms of the control voltage and the emitted current. In the linac, the required electron beam current is relatively small and the beam energy is low. A large beam aperture can be used and results in a small current density. In this case, a linear approximation of the emitted current in Eq. 5 can be used. $G_{\text {emit }}$ is the emission coefficient which is in the range of $10-30 \mathrm{~mA} / \mathrm{V}$ for practical cathodes. $U_{\text {cutoff }}$ describes the cutoff voltage which can be obtained from previously published results [21].

$$
I=G_{\text {emit }}\left(U_{\text {grid }}-U_{\text {cutoff }}\right)
$$
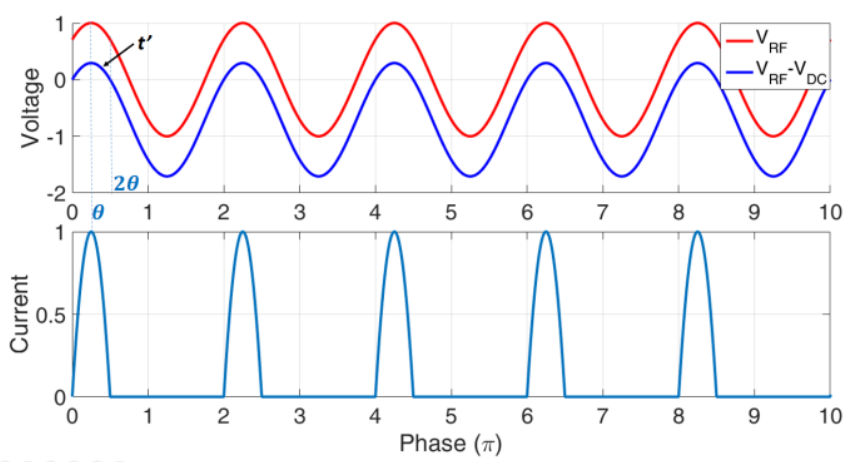

Fig. 3 The driving voltage and the emitted current

The bunch charge, bunch length and emittance are important parameters of the RF gun in the linac. The bunch charge can be integrated from the emitted current and is

$$
Q=\int I(t) d t=\frac{U_{r f}}{f} \frac{2 G_{e m i t}}{2 \pi}(\sin \theta-\theta \cos \theta)
$$

where $\cos \theta=\left(-U_{\text {bias }}+U_{\text {cutoff }}\right) / U_{r f}$ denotes when the electrons are allowed to be emitted. The bunch length of the emitted current is determined by the phase $\theta$ with $\tau=\theta / 2 \pi f$.

The electrons will travel to the grid under the applied electric field. To simplify the calculation, the space charge effect is ignored and the electric field between the emitter and the grid is regarded as a uniform distribution, as shown in Fig. 2. The electron's longitudinal position follows Eq. 7.

$$
\ddot{L}(t)=\frac{e}{m} \frac{U_{\text {grid }}}{d}
$$

The electrons may experience both accelerating and decelerating electric fields. Only the electrons emitted before time $t^{\prime}$ is able to pass through the grid and escape the cathodegrid region to the anode. Otherwise they will be reflected back to the emitter by the decelerating electric field. The time $t^{\prime}$ determines the bunch duration from the emitter, which is defined by Eq. 8 .

$$
L(2 \tau)-L\left(\mathrm{t}^{\prime}\right)=d
$$

It is preferred to have $t^{\prime}$ close to $2 \tau$ to reduce the beam current lost, since the backstreaming electrons will reduce the lifetime of the cathode. In this case, the gap distance $d$ should be as small as possible. However, it has a physical constraint of about $0.16 \mathrm{~mm}$. The gap length also sets a limit to the voltage between the cathode and the grid, which is $U_{r f}+\left|U_{\text {bias }}\right|$, due to the vacuum breakdown.

The theoretical model provides the emission process of the electron beam, as well as the relationship between the electron bunch length, bunch charge, and the applied RF and bias voltages. The bottom threshold of the emitted bunch length for a given bunch charge can be obtained by solving the Eq. 4-8. Fig. 4 shows the results at $1.5 \mathrm{GHz}$ and $3.0 \mathrm{GHz}$, respectively. In the calculation, the gap distance of $0.16 \mathrm{~mm}$ was used. A reasonable emission coefficient $G_{\text {emit }}$ of $18 \mathrm{~mA} / \mathrm{V}$, and the limit of $U_{r f}+\left|U_{\text {bias }}\right|=600 \mathrm{~V}$ was chosen. With a bunch charge of $33.3 \mathrm{pC}$ at $1.5 \mathrm{GHz}$, the bunch length is about $68 \mathrm{ps}$ when $\theta=40^{\circ}$. With a bunch charge of $16.7 \mathrm{pC}$ at $3.0 \mathrm{GHz}$, the bunch length is about $23 \mathrm{ps}$ when $\theta=45^{\circ}$. For this idealised case operating at higher frequency helps to reduce the emitted bunch length.

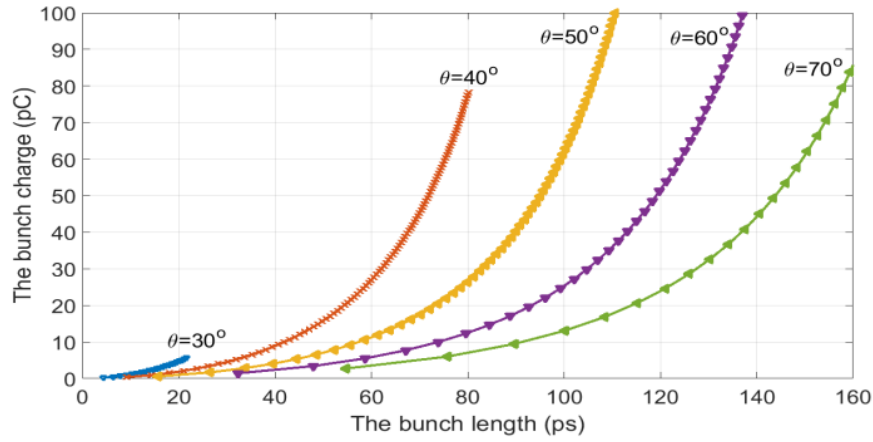

(a)

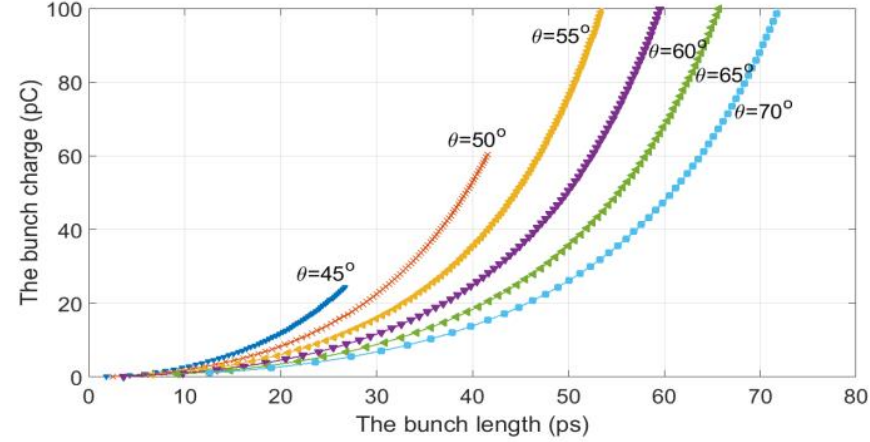

(b)

Fig. 4. The relation between bunch length and bunch charge at different operating frequencies, (a) $1.5 \mathrm{GHz}$, and (b) $3.0 \mathrm{GHz}$. 


\section{DESIGN OF THE ELECTRON GUN GEOMETRY}

The theoretical analysis does not include the effects such as the space charge, thermal emission distribution and the beam dynamics between the grid and anode. In this section, a practical electron gun is designed. The performance was investigated through numerical particle-in-cell simulations.

The design of the RF gun is based on the Pierce-type configuration which is commonly used in the conventional vacuum electronic devices, such as traveling wave tubes, klystrons and inductive output tubes (IOTs) [22]. The initial geometry was generated based on the Vaughan synthesis [23] with 4 input parameters, including the beam voltage, beam current, emitter radius as well as the beam waist, as listed in Table 1 . The initial geometry was then used as the starting point for further optimization to get more accurate simulation results and to further improve the electron beam quality, including good laminarity and a small emittance. Ideally, the finitedifference-time-domain method and particle-in-cell (FDTDPIC) simulation which is able to simulate the beam dynamics when driven by RF field is required. However, it is timeconsuming as a fine mesh has to be used to represent the thin grid structure. In the optimization, it was more practical to use 2D DC-voltage gun design code EGUN to simulate the electron beam trajectory since only $5 \%$ of the simulation time was required. The optimal geometry was then applied with the grid structure to investigate further beam dynamics with the RF driving field using the FDTD-PIC code MAGIC.

The Pierce-type gun is normally with a curved emitter surface to achieve a large beam compression ratio, however, it is not a critical parameter of the required RF gun since a large beam radius is acceptable. The emitter surface was therefore simplified by using a readily available, cost-effective, commercial gridded cathode. The model chosen was the NJK2221 A gridded thermionic cathode from Japan Radio Co. Ltd. It has a flat emission surface with a radius of $4.0 \mathrm{~mm}$, and a grid distance of $0.16 \mathrm{~mm}$. The flat emitter surface also helped to save the simulation time since a less dense mesh grid could be used to represent a flat surface as compared to a curved cathode surface.

The beam trajectories of the optimized geometry simulated by EGUN are shown in Fig. 5(a). The acceleration voltage between the cathode and anode was $25 \mathrm{kV}$ and the emitted current was 1.0 A. The radius of the beam waist was $\sim 1.5 \mathrm{~mm}$. The beam trajectories ware exported for post-processing the emittance. The root mean square (RMS) normalized transverse beam emittance defined by $\varepsilon_{\text {rms }}=\sqrt{\left\langle x^{2}\right\rangle\left\langle x^{\prime 2}\right\rangle-\left\langle x x^{\prime}\right\rangle^{2}}$ at the exit of the anode was $1.0 \mathrm{~mm} \cdot \mathrm{mrad}$, where $x$ and $x^{\prime}$ were the position and angle of the electrons in $x$ coordinate [24]. Its geometry was then simulated with MAGIC. The complete configuration including the input cavity described in section II together with the grid structure is shown in Fig. 5(b). When the gun is only driven by the DC acceleration voltage without the $\mathrm{RF}$ voltage, the simulation results are the same as EGUN. When driven by an RF voltage and a bias voltage, the electron bunch was generated. Fig. 6 shows the spatial distribution of electrons at different time frames, corresponding to the process of bunch emission from the emitter, bunch acceleration by the electric field and at the exit of the anode.

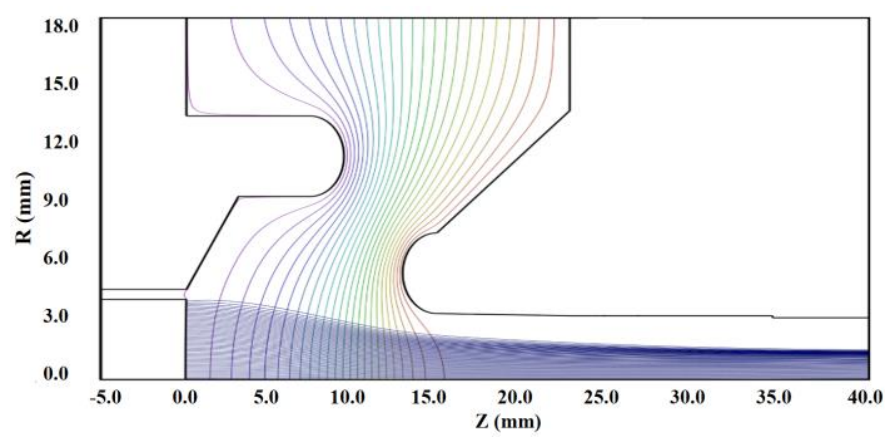

(a)

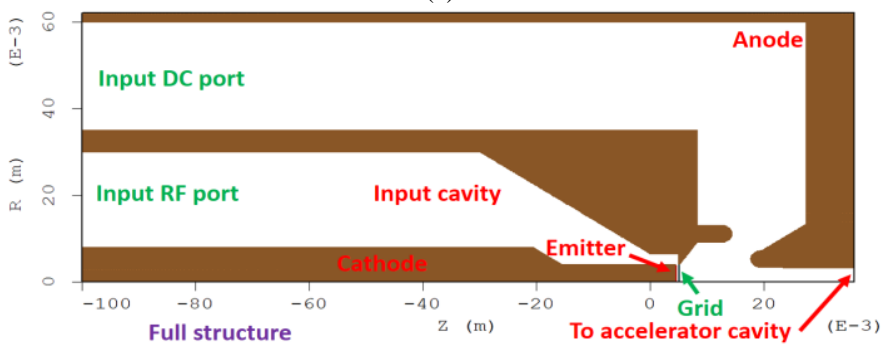

(b)

Fig. 5 The optimized geometry of the gun from EGUN (a) and the model of the $\mathrm{RF}$ gun including the input coupler used in simulations with MAGIC (b).

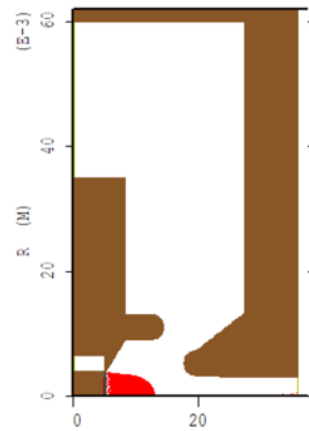

(a)

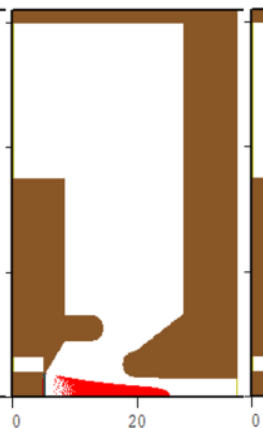

(b)

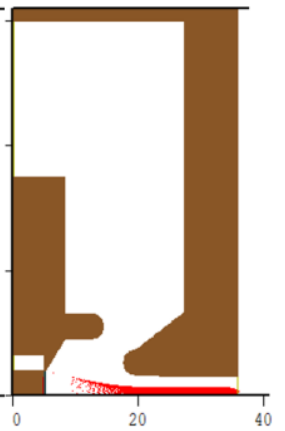

(c) $\quad z \quad(M)$
Fig. 6 The spatial distribution of electrons at different simulation time frames, (a) bunch emitted from the emitter, (b) bunch acceleration by the electric field, (c) bunch at the exit of the anode.

\section{PERFormanCE OF THE RF GUN}

The performance of the RF gun was studied by applying different combinations of the RF and bias voltages. The simulated results of the peak emitted current as a function of the applied voltage are shown in Fig. 7. At different DC bias voltages, the curves are close to each other at the same total voltages $U_{\text {grid }}=U_{r f}+U_{\text {bias }}$. The results show good linearity of the emitted current over a wide range of the grid voltages, which validates the approximation of Eq. 4 . The value of $G_{\text {emit }}$ from the linear fit was about $18 \mathrm{~mA} / \mathrm{V}$.

Fig. 8 shows the emitted current from the cathode and the collected current at the entrance of the acceleration cavity at $U_{r f}=170 \mathrm{~V}$ and $U_{\text {bias }}=-115 \mathrm{~V}$. The waveform of the emitted current matched well with the theoretical prediction. The amplitude of the collected current pulse waveform of the emitted current was about $40 \%$ of the emitted current. The charge in the individual collected and emitted current pulse was 
calculated by integrating the current and they were found to be $72 \mathrm{pC}$ and $43 \mathrm{pC}$, respectively. The ratio between them was about $60 \%$, which indicated that $40 \%$ of the electrons emitted from the cathode backstreamed to the emitter surface due to the deceleration voltage. The PIC simulation shows a much larger value than the theoretical prediction.

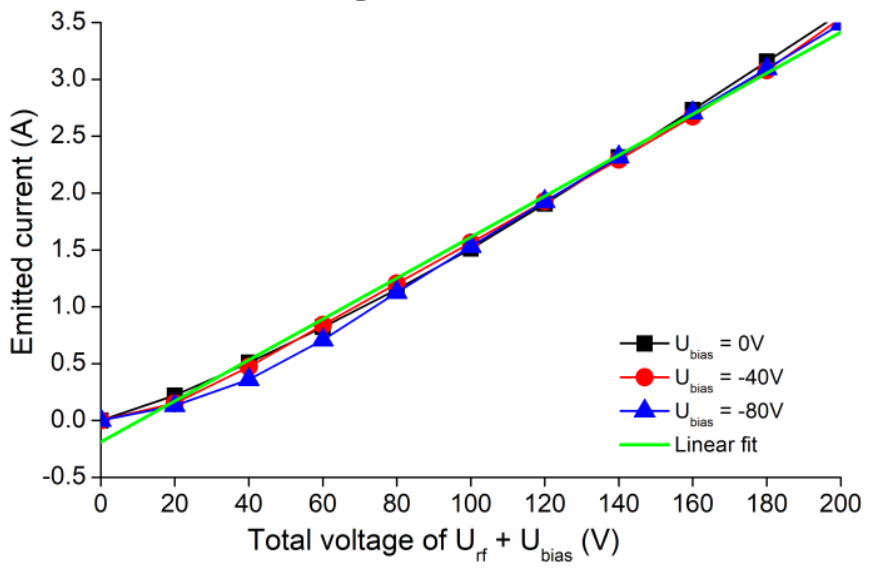

Fig. 7 The relationship between the grid voltage and the emitted current.

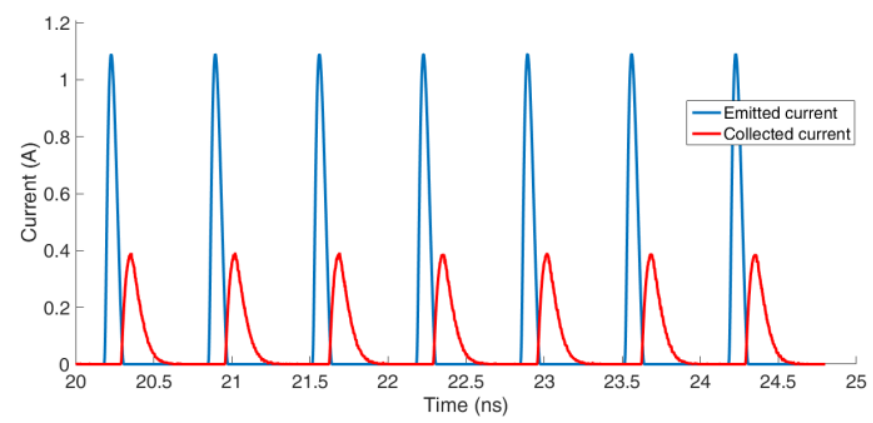

Fig. 8 The emitted current from the cathode and the collected current at the entrance of the accelerator cavity.

The Full-width-half-maximum (FWHM) lengths of the emitted and collected bunches were 72 ps and 107 ps respectively from the simulation. It is much larger than the theoretical prediction. The theoretical model did not include the effect of space charge at the emitter, thermal emission distribution and the beam dynamics between the grid and anode. Further simulations were carried out to identify and quantify these factors which resulted in the widening of the bunch. they include:

(1) Velocity differences due to the different grid voltages as a function of the time. The head of the bunch had larger energy and the tail had less energy when driven by the RF field. Considering electrons accelerated by grid voltages $30 \mathrm{~V}$ and $10 \mathrm{~V}$, the time difference was $81 \mathrm{ps}$ with a traveling distance of $0.36 \mathrm{~mm}$ (the grid distance plus the grid thickness) in the PIC simulation which takes into account the space charge at the emitter. It is the major reason for the widening of the pulse.

(2) Different path lengths of the electrons. The electrons emitted from different positions traveled with different paths. The electrons emitted from larger emitter radius travel longer distances. The difference in the traveling time from the EGUN simulation was $28 \mathrm{ps}$.

(3) The space charge force accelerates the head of the bunch and decelerates the tail of the bunch. EGUN simulations show there was about $16 \mathrm{ps}$ additional contribution to the pulse length due to space charge effects. The space charge force would have less impact when operating at a smaller beam current.

The sliced transverse emittance was also calculated by statistically counting the electrons at the exit of the anode. Fig. 9 shows the correlated emittance and the current of the electron bunch as the function of the simulation time. The emittance was lower at the head of the bunch and it increased as a function of the bunch charge. The major part of the bunch had an emittance of less than $17 \mathrm{~mm} \cdot \mathrm{mrad}$. The tail of the bunch has larger emittance however the current contained in this part of the bunch was small. The noise of the emittance at the end of the bunch was due to the unstable statistical variation due to the small number of electrons. The PIC simulation showed a much bigger emittance compared to the static electric field case. The relatively weak acceleration field due to the RF voltage applied between the cathode and grid, as well as the uneven space charge force on the bunch were the main reasons for the high emittance of the RF gated gun.

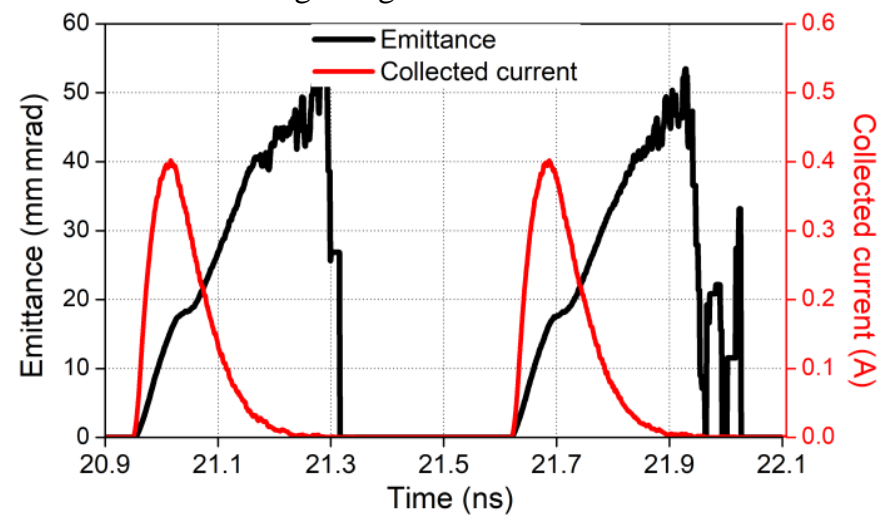

Fig. 9 The emittance of the electron at the exit of the anode.

By varying the RF and bias voltage, the bunch length and bunch charge of the collected current change accordingly. Fig. 10(a) shows the simulation results at different combinations of $U_{r f}$ and $U_{\text {bias }}$ at a driven frequency of $1.5 \mathrm{GHz}$. When fixing $U_{\text {bias }}$ while increasing $U_{r f}$ both larger bunch charge and longer bunch length were observed. However, the growing of the bunch charge as a function of bunch length follows a linear trend, which was different from the theoretical prediction shown in Fig. 4. The theoretical model showed that smaller $\theta$ (equivalently larger $U_{\text {bias }}$ value) helps to reduce the bunch length. The PIC simulations also show the same trend. However, its impact on the bunch length was very small. By increasing the bias voltage from $-80 \mathrm{~V}$ to $-170 \mathrm{~V}$, the difference in the bunch length was about 5 ps only at the same bunch charge. The minimum bunch length of 106 ps could be achieved with a bunch charge of $33.3 \mathrm{pC}$.

The average transverse emittances at different RF and bias voltages were also studied. When fixing $U_{\text {bias }}$ while increasing $U_{r f}$, a higher grid voltage can be achieved and it helped to reduce the transverse emittance. A minimum transverse emittance of $12 \mathrm{~mm} \cdot \mathrm{mrad}$ could be achieved. At different bias voltages, the trend of transverse emittance was similar. Since the DC acceleration region was also a main source of the 
emittance, it is possible to improve the emittance by further optimizing the geometry of the cathode and anode.

Fig. 4 shows that the length of the emitted pulse can be reduced by operating at the higher RF frequency. The PIC simulation proved the trend, however, it was found that more electrons were backstreamed to the emitter when operating at a higher frequency. The bunch length and bunch charge at different grid voltages with RF frequency of $3.0 \mathrm{GHz}$ are shown in Fig. 10(b). The minimum bunch length of $100 \mathrm{ps}$ could be achieved with a bunch charge of $16.7 \mathrm{pC}$, which means the bunch length at the entrance of the linac was reduced slightly at a higher frequency. Since the first two reasons for the pulse widening were mainly determined by the electron gun structure. In this case no significant advantage was to be gained by operating at higher RF frequency as this does not reduce the pulse width or shortens the bunch length. When operating at a higher frequency, the emitted current had to have larger compensation due to the higher ratio of the backstreaming electrons. The space charge force, therefore, became larger and resulted in longer pulse widening. It also results in larger emittance.

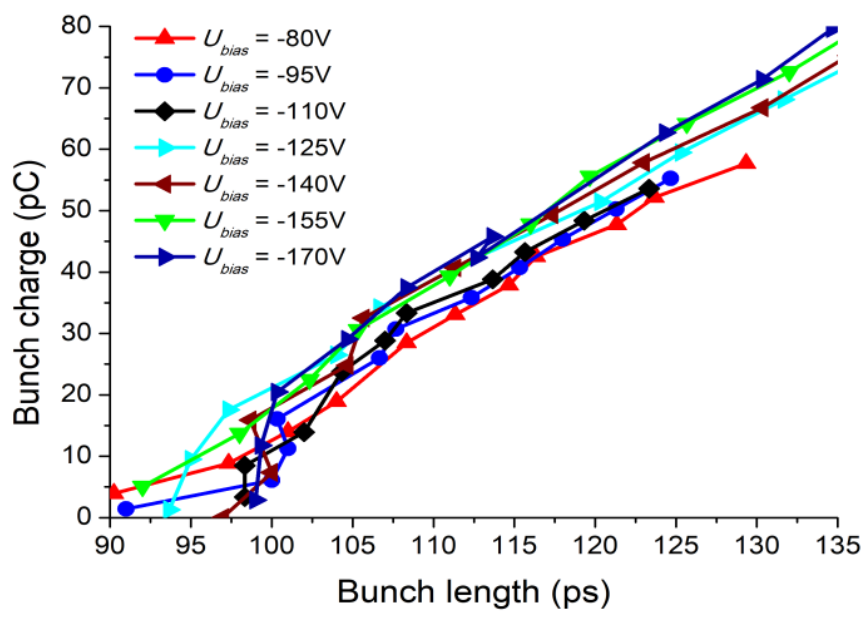

(a)

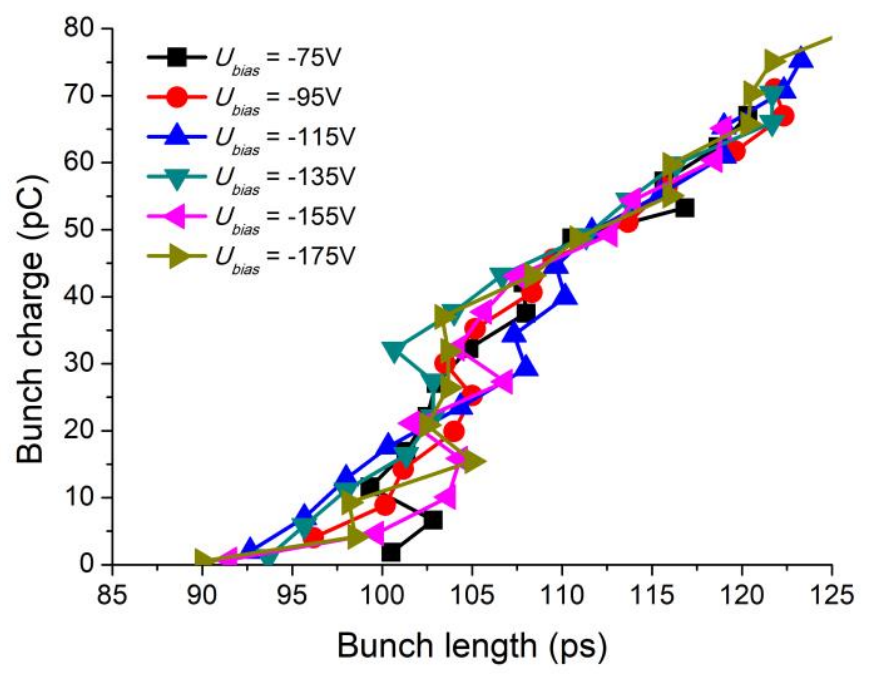

(b)

Fig. 10 The relationship between the bunch length and bunch charge at different grid voltages with RF frequency of (a) $1.5 \mathrm{GHz}$ and (b) $3.0 \mathrm{GHz}$.

\section{CONCLUSION}

In this paper, a thermionic RF gun for particle accelerator applications was investigated. The electron gun was based on a commercially available gridded cathode and the geometry was optimized based on the Pierce-type configuration. A theoretical model was used to predict the relationship between the bunch length and charge. However, it is limited by ignoring the effect of space charge at the emitter and the beam dynamics between the grid and anode. The performance of the electron gun was further studied by PIC simulations. The reasons for the pulse widening have been analyzed.

The minimum pulse length of 106 ps could be achieved with a bunch charge of $33.3 \mathrm{pC}$ when the driving RF frequency was $1.5 \mathrm{GHz}$. The average transverse emittance was about 17 $\mathrm{mm} \cdot \mathrm{mrad}$. Increasing the driven RF frequency helps to reduce the pulse length of the emitted current. However, the improvement to the collected current was not significant. The velocity spread due to the different grid voltages as the function of the time is the main reason for the widening of the pulse from the emitted current to the collected current at the entrance of the acceleration cavity. It is not dependent on the driving RF frequency and is mainly determined by the grid distance and thickness of the grid structure.

Compared with the photocathode injection gun, the emittance of the proposed RF gun is much larger, which limits its applications in linacs that can operate with electron pulses of a certain brightness. However, by combining the merits of compact, demountable, and long lifetime, applications can be found in medical and industry linacs where the ultimate brightness is not required.

\section{REFERENCES}

Z. Huang and K.-J. Kim, "Review of x-ray free-electron laser theory," Physical Review Special Topics - Accelerators and Beams, vol. 10, no. 3, p. 034801, March 2007. DOI: 10.1103/PhysRevSTAB.10.034801

J. St. Aubin, S. Steciw, C. Kirkby, and B. G. Fallone, "An integrated $6 \mathrm{MV}$ linear accelerator model from electron gun to dose in a water tank," Med. Phys., vol. 37, no. 5, pp. 2279-2288, 2010. DOI: $10.1118 / 1.3397455$

C. Mitchell, P. Sprangle, and J. Peñano, "A Gridded Thermionic Injector Gun for High-Average-Power Free-Electron Lasers," IEEE Trans. Plasma Sci., vol. 40, no. 8, pp. 1977-1983, 2012. DOI: 10.1109/TPS.2012.2201962

D. H. Dowell, K. J. Davis, K. D. Friddell, E. L. Tyson, C. A. Lancaster, L. Milliman, R. E. Rodenburg, T. Aas, M. Bemes, S. Z. Bethel, P. E. Johnson, K. Murphy, C. Whelen, G. E. Busch, and D. K. Remelius, "First operation of a photocathode radio frequency gun injector at high duty factor," Appl. Phys. Lett., vol. 63, no. 15, pp. 2035-2037, 1993/10/11 1993. DOI: 10.1063/1.110583

C.-M. Tang, A. C. Ting, and T. Swyden, "Field-emission arrays a potentially bright source," Nucl. Instrum. Methods. Phys. Res. A, vol. 318, no. 1, pp. 353-357, 1992/07/01/ 1992. DOI: https://doi.org/10.1016/0168-9002(92)91080-S

X. J. Wang, X. Qiu, and I. Ben-Zvi, "Experimental observation of high-brightness microbunching in a photocathode rf electron gun," Physical Review E, vol. 54, no. 4, pp. R3121-R3124, 10/01/ 1996. DOI: 10.1103/PhysRevE.54.R3121

I. V. Bazarov and C. K. Sinclair, "Multivariate optimization of a high brightness dc gun photoinjector," Physical Review Special Topics - Accelerators and Beams, vol. 8, no. 3, p. 034202, 03/24/ 2005. DOI: 10.1103/PhysRevSTAB.8.034202

P. Muggli, V. Yakimenko, M. Babzien, E. Kallos, and K. P. Kusche, "Generation of Trains of Electron Microbunches with Adjustable 
Subpicosecond Spacing," Phys. Rev. Lett., vol. 101, no. 5, p. 054801, 07/29/ 2008. DOI: 10.1103/PhysRevLett.101.054801

[9] B. Dunham, J. Barley, A. Bartnik, I. Bazarov, L. Cultrera, J. Dobbins, G. Hoffstaetter, B. Johnson, R. Kaplan, S. Karkare, V. Kostroun, Y. Li, M. Liepe, X. Liu, F. Loehl, J. Maxson, P. Quigley, J. Reilly, D. Rice, D. Sabol, E. Smith, K. Smolenski, M. Tigner, V. Vesherevich, D. Widger, and Z. Zhao, "Record high-average current from a high-brightness photoinjector," Appl. Phys. Lett., vol. 102, no. 3, p. 034105, 2013/01/21 2013. DOI: 10.1063/1.4789395

[10] K. Togawa, T. Shintake, T. Inagaki, K. Onoe, T. Tanaka, H. Baba, and H. Matsumoto, "CeB6 electron gun for low-emittance injector," Physical Review Special Topics - Accelerators and Beams, vol. 10, no. $2, \quad$ p. 020703 , 02/02/ 2007. DOI: 10.1103/PhysRevSTAB.10.020703

[11] A. Opanasenko, V. Mytrochenko, V. Zhaunerchyk, and V. A. Goryashko, "Design study of a low-emittance high-repetition rate thermionic rf gun," Physical Review Accelerators and Beams, vol. 20, no. 5, p. 053401, 05/31/ 2017. DOI: 10.1103/PhysRevAccelBeams.20.053401

[12] S. Suemine, K. Kawase, N. Sugimoto, S. Kashiwagi, K. Furukawa, R. Kato, A. Irizawa, M. Fujimoto, H. Ohsumi, M. Yaguchi, S. Funakoshi, R. Tsutsumi, K. Kubo, A. Tokuchi, and G. Isoyama, "Grid pulser for an electron gun with a thermionic cathode for the high-power operation of a terahertz free-electron laser," Nucl. Instrum. Methods. Phys. Res. A, vol. 773, pp. 97-103, 2015/02/11/ 2015. DOI: https://doi.org/10.1016/j.nima.2014.10.071

[13] S. H. Gold, A. Ting, V. Jabotinski, B. Zhou, and P. Sprangle, "Development of a high average current rf linac thermionic injector," Physical Review Special Topics - Accelerators and Beams, vol. 16, no. 8, p. 083401, 08/01/ 2013. DOI: 10.1103/PhysRevSTAB.16.083401

[14] P. Sprangle, J. Peñano, B. Hafizi, D. Gordon, S. Gold, A. Ting, and C. Mitchell, "High average current electron guns for high-power free electron lasers," Physical Review Special Topics - Accelerators and Beams, vol. 14, no. 2, p. 020702, 02/14/ 2011. DOI: 10.1103/PhysRevSTAB.14.020702

[15] E. H. Zubizarreta, E. Fidarova, B. Healy, and E. Rosenblatt, "Need for Radiotherapy in Low and Middle Income Countries - The Silent Crisis Continues," Clinical Oncology, vol. 27, no. 2, pp. 107-114, 2015/02/01/ 2015. DOI: https://doi.org/10.1016/j.clon.2014.10.006

[16] B. Whelan, L. Holloway, D. Constantin, B. Oborn, M. BazalovaCarter, R. Fahrig, and P. Keall, "Performance of a clinical gridded electron gun in magnetic fields: Implications for MRI-linac therapy," (in eng), Med. Phys., vol. 43, no. 11, pp. 5903-5903, 2016. DOI: $10.1118 / 1.4963216$

[17] K. Torgasin, K. Mishima, H. Zen, K. Yoshida, H. Negm, M. Omer, T. Kii, K. Nagasaki, K. Masuda, and H. Ohgaki, "Properties of quarter-wavelength coaxial cavity for triode-type thermionic RF gun," Jpn. J. Appl. Phys., vol. 56, no. 9, p. 096701, 2017/08/04 2017. DOI: $10.7567 /$ jjap. 56.096701

[18] M. Reiser, Theory and design of charged particle beams. 1995.

[19] R. Vaughan, "A synthesis of the Longo and Eng cathode emission models," IEEE Trans. Electron Devices, vol. 33, no. 11, pp. 19251927, 1986. DOI: 10.1109/T-ED.1986.22844

[20] L. K. Ang and P. Zhang, "Ultrashort-Pulse Child-Langmuir Law in the Quantum and Relativistic Regimes," Phys. Rev. Lett., vol. 98, no. $16, \quad$ p. $164802, \quad 04 / 19 / 2007 . \quad$ DOI: 10.1103/PhysRevLett.98.164802

[21] R. J. Bakker, C. A. J. van der Geer, A. F. G. van der Meer, P. W. van Amersfoort, W. A. Gillespie, and G. Saxon, "1 GHz modulation of a high-current electron gun," Nucl. Instrum. Methods. Phys. Res. $A$, vol. 307, no. 2, pp. 543-552, 1991/10/01/ 1991. DOI: https://doi.org/10.1016/0168-9002(91)90229-J

[22] A. S. Gilmour, Klystrons, traveling wave tubes, magnetrons, crossed-field amplifiers, and gyrotrons. Artech house, 2011.

[23] J. R. M. Vaughan, "Synthesis of the Pierce gun," IEEE Trans. Electron Devices, vol. 28, no. 1, pp. 37-41, 1981. DOI: 10.1109/TED.1981.20279

[24] K. Floettmann, "Some basic features of the beam emittance," Physical Review Special Topics - Accelerators and Beams, vol. 6, no. 3 , p. 034202, 03/06/ 2003. DOI: 10.1103/PhysRevSTAB.6.034202 